\title{
Use of ulipristal acetate and levonorgestrel for emergency contraception: a follow-up study
}

\author{
Aisling Susan Baird, ${ }^{1}$ James Trussell, ${ }^{2}$ Anne Webb $^{3}$
}

\begin{abstract}
${ }^{1}$ Consultant in Sexual and Reproductive Health, Abacus Community Sexual Health Service, Liverpool Community Health, Liverpool, UK ${ }^{2}$ Professor of Economics and Public Affairs, Office of Population Research, Princeton University, Princeton, NJ, USA and Visiting Professor, Hull York Medical School, University of Hull, Hull, UK

${ }^{3}$ Retired Consultant in Sexual and Reproductive Health, Abacus Community Sexual Health Service, Liverpool Community Health, Liverpool, UK
\end{abstract}

\section{Correspondence to}

Dr Aisling Susan Baird, Abacus Community Sexual Health Service, Liverpool Community Health, The BEAT, David Lewis Street, Liverpool L1 4AP, UK; aisling.baird@liverpoolch.nhs.uk

Received 22 July 2013 Revised 11 March 2014 Accepted 23 April 2014 Published Online First 28 May 2014

CrossMark

To cite: Baird AS, Trussell J, Webb A. J Fam Plann Reprod Health Care 2015;41:

116-121.

\begin{abstract}
Objectives Previously we showed that increasing the choice of emergency contraception (EC) guided by medical eligibility did not result in wholesale large-scale usage of ulipristal acetate (UPA). This further 12-month study aimed to answer three questions. (1) Does offering choice of EC lead to change in methods used? (2) Are women who choose UPA more likely than those who choose levonorgestrel (LNG) to continue using condoms for subsequent contraception or to decline any ongoing contraception? (3) Do more women choosing LNG 'quick start' hormonal contraception? Methods A retrospective study of EC episodes (1 April 2012 to 31 March 2013) by quarters. Among women offered all three methods of EC (49.1\%) we noted the method chosen, and decisions on ongoing contraception among those choosing either LNG or UPA. Differences were tested for statistical significance.

Results In 6110 episodes of EC, LNG was issued in $69.2 \%$, UPA in $26.0 \%$, and a copper intrauterine device (Cu-IUD) was fitted in $4.8 \%$. Quarter by quarter, the data show a small decline in LNG use, suggesting plateauing by the last quarter, and a significant increase in UPA use between the first and the other three quarters $(p<0.001)$. Use of the CU-IUD remained static. The percentage of women offered three methods rose to $54.2 \%$. In women offered full choice $(3000 ; 49.1 \%)$ we saw a significant increase in choice of UPA, from $39.3 \%$ to $48.6 \%(p<0.001)$. Women who chose LNG were more likely to quick start $(p=0.02)$ or be continuing contraception already used $(p<0.001)$. Overall, those choosing UPA were more likely to use condoms $(p<0.001)$ but were no more likely to decline ongoing contraception ( $p=0.13$ ).
\end{abstract}

Conclusions There was a significant increase in women using UPA for EC compared with our previous study, particularly among those wishing to use condoms for continuing contraception. Women choosing LNG were more likely to quick

\section{Key message points}

- One year following the introduction of new Faculty of Sexual \& Reproductive Healthcare guidance, more women were offered all three available methods of emergency contraception.

- Many women continued to choose levonorgestrel (LNG) when offered full choice.

- Those women who chose ulipristal acetate more often continued contraception with condoms and those who choose LNG mostly 'quick started' hormonal contraception.

start pills or to continue current hormonal contraception. Detailed attention to continuing contraception following EC may be an important factor in the prevention of unwanted pregnancy.

\section{INTRODUCTION}

In a previous study we examined the effect of the introduction of new emergency contraception (EC) guidance from the UK Faculty of Sexual \& Reproductive Healthcare (FSRH) in Liverpool and Knowsley, UK. ${ }^{1}$ The guidance was introduced in 2011 and recommended that all women requesting EC should have their individual needs assessed and be informed of the available methods, efficacy, adverse effects, interactions, eligibility and additional contraception. ${ }^{2}$ The more costly ulipristal acetate (UPA) has been shown to be active for longer during the days of the cycle when pregnancy risk is highest, around the time of the luteinising hormone (LH) surge. $^{3}$ This finding backs up its superior efficacy seen in meta-analysis of clinical studies. ${ }^{4}$ 
Previously, we studied two 3-month periods of EC requests, immediately prior to and following the adoption of the new FSRH guidance. The use of levonorgestrel (LNG) fell from $93 \%$ of EC to $76 \%$. The use of UPA rose from $3.0 \%$ to $18.7 \%$ and the use of the copper intrauterine device (Cu-IUD) remained about the same. We also found that in some cases only LNG was offered, and that in a large percentage of these such action was appropriate. We postulated that if offered all three methods, many women would opt for LNG because they were familiar with it and wished to' quick start' or continue their current hormonal method of contraception, with a shorter period of requirement for additional condom use. 'Quick starting' refers to starting hormonal contraception on the same day or the day after taking oral EC rather than waiting until the next menstrual period. This is recommended by the FSRH as oral EC does not work prospectively and further intercourse in the same cycle has been associated with a higher pregnancy risk. ${ }^{5}$ Current UK guidance suggests that following quick starting, extra contraceptive precautions should be taken for 7 days longer after using UPA than after LNG. ${ }^{6}$

We suggested that the situation be studied again after a year to see whether greater staff familiarity with the FSRH guidance or greater patient familiarity with UPA resulted in a different pattern of use.

The present study assessed what happened to UPA use over 12 months and whether the choice of intended method of contraception following EC varied with the choice of oral EC type (LNG vs UPA). Our study questions were:

- After full choice training was implemented, did choices offered for EC change with time?

- Do more women choose UPA when they plan either to continue using condoms for ongoing contraception or if they decline any ongoing contraceptive method?

- Do more women choosing LNG either start ongoing contraception by 'quick starting' hormonal contraception or continue with their current hormonal method?

Our service has been described previously. ${ }^{1}$ In brief, a population total of about 600000 is served in a multiple-site service, with over 25 clinical delivery sites. The majority of this service delivery, including EC provision, is undertaken by registered practitioners with a nursing or midwifery background, only a few of whom fit Cu-IUDs. Specialist medical practitioners are available for advice but are not present at every site; although this barrier could affect the provision of $\mathrm{Cu}-\mathrm{IUD}$ fitting, processes for rapid referral are available.

\section{METHODS}

We retrospectively studied all EC episodes from 1 April 2012 to 31 March 2013 by interrogating
Excelicare $^{\mathrm{TM}}$ (AxSys Technology Ltd, Paisley, UK), our electronic patient record (EPR) system. We counted the total number of EC episodes and broke these down into quarterly time periods. Then, among women offered all three methods of EC we noted the eventual method chosen by the woman. Finally, again among women offered all three methods of EC, the decisions of those choosing LNG and those choosing UPA regarding ongoing contraception were determined. The decisions were grouped into seven categories: continuing current hormonal method, quick starting, starting hormonal contraception with the next menstrual period, using condoms as a sole method, 'other method' (e.g. going to see a general practitioner to discuss ongoing methods or continuing with diaphragm use); postcoital Cu-IUD fitting; declining contraception altogether. Some women were not able to access a $\mathrm{Cu}$-IUD at their initial visit despite this being their first choice. They have been included in one of the oral methods as they were issued LNG or UPA pending attendance at another site. We defined 'current' in 'continuing current hormonal method' as being within 1 week of existing hormonal contraception no longer being effective, according to national recommendations. This meant that we used the following cut-off points:

- Within 14 days of last active combined hormonal contraceptive dose

- Within 7 days of last progestogen-only oral contraceptive pill or implant removal

- Less than 15 weeks since the last dose of depot medroxyprogesterone acetate.

There was a small number of 'duplicates' due to the way in which the EPR interprets a request. They did not affect the overall outcome of the analysis.

Statistical analysis was undertaken using StatXact ${ }^{\mathrm{TM}}$ software (Cytel, Cambridge, MA, USA). For comparison of method of subsequent contraception or no contraception between those choosing UPA or LNG, and change of usage with time, we used Fisher's exact test.

\section{RESULTS}

In the 12 calendar months from April 2012 to March 2013 there were 6110 episodes of EC. About $60 \%$ of episodes overall were undertaken at a clinical site where there was no $\mathrm{Cu}-\mathrm{IUD}$ fitter present. Most of these were accounted for by the approximately 50\% of episodes seen at a city centre service at which no practical procedures are performed but which is 10 minutes' walk from a larger clinical site where all procedures are available. Doctors saw 13\% of women, compared with $8 \%$ in our previous study. In 4228 (69.2\%) consultations LNG was issued, in 1589 (26.0\%) UPA and in 293 (4.8\%) the episode resulted in the fitting of a Cu-IUD. There is a quarterly decline in the use of LNG, with a suggestion of plateauing out by the last quarter, and a significant increase in the use of UPA between the first quarter and the other 
Table 1 Emergency contraception use by quarter from April 2012 to March 2013

\begin{tabular}{|c|c|c|c|c|c|}
\hline Type of EC used & April-June [n (\%)] & July-September [n (\%)] & October-December [n (\%)] & January-March [n (\%)] & Total $[n(\%)]$ \\
\hline$\overline{L N G}$ & $1099(75.3)$ & $1079(69.9)$ & $1016(67.3)$ & $1034(64.7)$ & $4228(69.2)$ \\
\hline UPA & $279(19.1)$ & $401(26.0)$ & $427(28.3)$ & $482(30.2)$ & $1589(26.0)$ \\
\hline Cu-IUD & $82(5.6)$ & $63(4.1)$ & $66 \quad(4.4)$ & $82(5.1)$ & $293(4.8)$ \\
\hline Total & 1460 & 1543 & 1509 & 1598 & 6110 \\
\hline
\end{tabular}

Cu-IUD, copper intrauterine device; EC, emergency contraception; LNG, levonorgestrel; UPA, ulipristal acetate.

three $(p<0.001)$. The rate of use of the Cu-IUD remained more or less static (Table 1 ).

Table 2 shows the documented methods of EC offered, by quarters. The figures for offering all three methods rose slightly in the last three quarters, reaching 866 (54.2\% of all offers) by the final quarter. Offers of five other combinations of methods remained approximately static, although in four (LNG and UPA, UPA and IUD, IUD alone and UPA alone) the numbers were too small to draw firm conclusions so they have been combined into one category. In 409 records $(6.7 \%$ of total EC episodes) no offered methods were documented.

Table 3 gives the choice of EC method in women who were offered all three options. We saw an increase in the choice of UPA from $229(39.3 \%)$ to $421(48.6 \%)$, and a corresponding decrease in the choice of LNG from 336 (57.6\%) to 423 (48.8\%). Most of these changes happened after the first 3 months. The increase in UPA use from the first quarter to the next three quarters was statistically highly significant $(p<0.001)$.

Table 4 shows the ongoing contraception methods planned by women who chose UPA and LNG after being given full choice. Women who chose LNG were more likely to quick start $(p=0.02)$ or to be continuing contraception already used $(p<0.001)$ than women who chose UPA. For either quick starting or continuing current hormonal method considered together, women choosing LNG were more likely to do this than those choosing UPA $(p<0.001)$. Among all women, those choosing UPA were more likely to use condoms $(p<0.001)$ but were no more likely to decline ongoing contraception $(p=0.23)$.
Of 2926 women offered full choice and taking either LNG or UPA, 1818 women wished to use ongoing hormonal contraception, of whom 1627 $(89.5 \%)$ elected to quick start or continue and 191 $(10.5 \%)$ chose to wait until their next period. Table 5 shows the intended methods of contraception chosen by women who wished to start with the next menstrual period.

\section{DISCUSSION}

This study shows how EC is offered and used in a large, open-access sexual and reproductive health service in the UK. One of its strengths is that it reflects a 'real world' scenario. Since our previous report we have seen an increase in the issue of UPA from just over $18 \%$ to $26 \%$ in a year (April 2012 to March 2013). In women offered all three methods of EC, the percentage choosing UPA rose from just under $40 \%$ to nearly $50 \%$. While UPA may have the benefit of increased contraceptive efficacy, ${ }^{3}{ }^{4}$ LNG remains the most frequently issued method of EC overall $(69.2 \%$ vs $26.0 \%$ ). However, the percentage using LNG is only slightly higher than that taking UPA $(51.3 \%$ vs $46.2 \%)$ in women offered all three methods of EC, and by the last quarter the proportions were nearly the same (48.6\% UPA vs $48.8 \%$ LNG). The accuracy of our data is dependent upon completeness of documentation in the EPR, particularly in the part of the record where choices given to the woman are documented. Overall we have documented evidence that at least $90.5 \%$ of women were offered LNG, $66.9 \%$ the IUD and $53.6 \%$ UPA. It is possible that the percentages were higher as $6.7 \%$ had no choices recorded in the tick boxes, which are not mandatory. However

Table 2 Documented methods of emergency contraception offered by quarter from April 2012 to March 2013

\begin{tabular}{|c|c|c|c|c|c|}
\hline Type of EC offered & April-June [n (\%)] & July-September [n (\%)] & October-December [n (\%)] & January-March [n (\%)] & Total $[n(\%)]$ \\
\hline LNG, UPA, Cu-IUD & $583(39.9)$ & $734(47.6)$ & $817(54.1)$ & $866(54.2)$ & $3000(49.1)$ \\
\hline LNG & $386(26.4)$ & $373(24.2)$ & $318(21.1)$ & $337(21.1)$ & $1414(23.1)$ \\
\hline LNG, CU-IUD & $302(20.7)$ & $263(17.0)$ & $202(13.4)$ & $183(11.5)$ & $950(15.5)$ \\
\hline Other combinations & $73(5.0)$ & $78 \quad(5.1)$ & $81 \quad(5.4)$ & 105 (6.6) & $337 \quad(5.5)$ \\
\hline Not documented & $116(7.9)$ & $95 \quad(6.2)$ & $91(6.0)$ & $107 \quad(6.7)$ & 409 (6.7) \\
\hline Total & 1460 & 1543 & 1509 & 1598 & 6110 \\
\hline
\end{tabular}

Cu-IUD, copper intrauterine device; EC, emergency contraception; LNG, levonorgestrel; UPA, ulipristal acetate.

NB. On the electronic patient records there is a tick box that asks what methods of EC have been offered, which is how these data were collated. However, this section is not mandatory and it relies on clinicians fully completing all sections of the patient record. It is usually possible to obtain these data by reading free-text sections; however, notes were not checked for this missing information. 
Table 3 Choice of emergency contraception method in women who were offered all three methods by quarter from April 2012 to March 2013

\begin{tabular}{|c|c|c|c|c|c|}
\hline Type of EC chosen & April-June [n (\%)] & July-September [n (\%)] & October-December [n (\%)] & January-March [n (\%)] & Total $[n(\%)]$ \\
\hline UPA & $229(39.3)$ & $355(48.4)$ & $382(46.8)$ & $421(48.6)$ & $1387(46.2)$ \\
\hline LNG & $336(57.6)$ & $365(49.7)$ & $415(50.8)$ & $423(48.8)$ & $1539(51.3)$ \\
\hline CU-IUD & $18 \quad(3.1)$ & 14 (1.9) & $20(2.4)$ & $22(2.5)$ & $74 \quad(2.5)$ \\
\hline Total & 583 & 734 & 817 & 866 & 3000 \\
\hline
\end{tabular}

Cu-IUD, copper intrauterine device; EC, emergency contraception; LNG, levonorgestrel; UPA, ulipristal acetate.

our previous study showed that amongst those women only offered LNG, nearly half (47.5\%) had clear reasons for this limited choice and 15\% did not have clear records. In the present study, fewer women were offered only LNG (23\% vs $33 \%$ previously) or had no choice recorded, suggesting that dissemination of the previous results to staff improved both record keeping and offering a choice of methods. The availability or otherwise of $\mathrm{Cu}$-IUD fitting at the time of request could also be a factor in a small number of cases. Women referred for fitting at an associated site could potentially default, and recognising this, the clinician offers oral EC at the initial visit. This would have affected our results because provision of an oral method (either LNG or UPA) for such women would have overstated requests for oral EC and women attending who had a Cu-IUD fitted will have been counted twice. Among women given full choice and wanting a $\mathrm{Cu}$-IUD but not being able to have it fitted at the time, more chose UPA (1.4\%) than LNG $(0.5 \%)$. However, these represent very small numbers.

Our study supports our previous postulation that greater familiarity with UPA by both women and clinicians would affect patient choice. The use of UPA increased significantly, but this change was mostly seen after the first 3 months, corresponding to the time soon after training occurred. Our training

Table 4 Ongoing contraception methods in women who chose levonorgestrel or ulipristal acetate after being offered the choice of all three emergency contraception methods

\begin{tabular}{|c|c|c|}
\hline $\begin{array}{l}\text { Contraceptive method } \\
\text { after EC pill }\end{array}$ & $\begin{array}{l}\text { LNG group } \\
{[n(\%)]}\end{array}$ & $\begin{array}{l}\text { UPA group } \\
{[n(\%)]}\end{array}$ \\
\hline Quick starting & $692(45.0)$ & $564(40.7)$ \\
\hline Condoms & $397(25.8)$ & $534(38.5)$ \\
\hline Continuing hormonal contraception & $285(18.5)$ & $86 \quad(6.2)$ \\
\hline $\begin{array}{l}\text { Starting hormonal contraception with } \\
\text { next period }\end{array}$ & $80 \quad(5.2)$ & $111(8.0)$ \\
\hline Declined ongoing contraception & 49 (3.2) & $56(4.0)$ \\
\hline Subsequent emergency Cu-IUD fitting & $7 \quad(0.5)$ & $20(1.4)$ \\
\hline Other method & $7(0.5)$ & $3(0.2)$ \\
\hline $\begin{array}{l}\text { Duplicate patient (not allocated to a } \\
\text { particular group) }\end{array}$ & $22(1.4)$ & $13(0.9)$ \\
\hline Total & 1539 & 1387 \\
\hline
\end{tabular}

Cu-IUD, copper intrauterine device; EC, emergency contraception; LNG, levonorgestrel; UPA, ulipristal acetate. emphasises that choice of EC should only be restricted by medical eligibility as recommended by FSRH guidance which states: "Health professionals should discuss individual need for ... [EC] and inform women about the different methods with regard to efficacy, adverse effects, interactions, medical eligibility and need for individual contraceptive precautions".

We do not recommend attempting to quantify the degree of pregnancy risk based on the calculated day of the cycle as the evidence does not support this. ${ }^{7-10}$

Our results endorse our previous conclusion that introduction of full availability of EC methods does not lead to a wholesale adoption of UPA, and our belief that in our service clinical staff only restrict choice where there are genuine medical eligibility issues. However, we acknowledge that, without perfect record keeping, which is not achievable in the real world, and manual checks of all records where full choice was apparently not given, it is not possible to prove this.

We were keen to understand better the contraceptive milieu around the choice of UPA versus LNG. We confirmed our hypothesis that in our service, if women are offered all three methods and choose an oral method, UPA is chosen in preference to LNG by women who wish to use condoms for continuing contraception, but refuted that the same happens in those who declined the offer of ongoing contraception. Another hypothesis was that for both quick starting or continuation of previous hormonal contraception, women choosing LNG were more likely to do either than those choosing UPA. The results support that hypothesis.

Glasier has been critical of the 'unlinking' of EC to continuing contraception by making EC more widely available to women by services in which further contraceptive provision is not undertaken. ${ }^{11}$ Wider availability of EC increases its usage but to date no effect on rates of unintended pregnancy has been seen. ${ }^{12}{ }^{13}$ A recent Cochrane update discusses the high rates of pregnancy observed in women who have additional episodes of sex in the same cycle as that in which EC was issued. ${ }^{5}$

An overwhelming majority of women in our service choose to quick start a hormonal contraception method following EC. Whether LNG or UPA was 
Table 5 Intended method of contraception chosen by women who were issued with levonorgestrel or ulipristal acetate after being given full choice of emergency contraception, and who chose to start with their next period

\begin{tabular}{lcc}
\hline Method & LNG group $(\boldsymbol{n})$ & UPA group $(\boldsymbol{n})$ \\
\hline COC & 56 & 71 \\
POP & 13 & 25 \\
CTP & 1 & 4 \\
SDI & $6^{*}$ & $5 \dagger$ \\
IUS & $4 \ddagger$ & $4 \ddagger$ \\
DMPA & 0 & 2 \\
Total & 80 & 111 \\
\hline
\end{tabular}

*Three women declined fitting at time of emergency contraception, although in all cases an implant could have been fitted; three were seen in a service that does not offer these procedures.

†All women seen in a service that does not offer these procedures. Al were bridged with condoms.

$\ddagger$ All used condoms in the interim.

COC, combined oral contraceptive pill; CTP, combined transdermal patch; DMPA, depot medroxyprogesterone acetate; IUS, intrauterine system; LNG, levonorgestrel; POP, progestogen-only pill; SDI, subdermal implant; UPA, ulipristal acetate.

chosen, very few women wished to wait until their next period to commence hormonal contraception. Our staff training emphasises the need to be nondirective about quick starting, preferring to offer women choice. Women themselves clearly choose to quick start if commencing hormonal contraception, or to continue it if already used. We suggest that if services fail to offer quick start, then their usage of UPA may increase as the difference in duration of need for extra precautions between LNG and UPA will disappear. Cameron and colleagues discuss the suggestion that there is a theoretical risk that UPA will interfere with the action of progestogen in hormonal contraceptives. There appears to be no difference between placebo and UPA on the chance of ovulation once the combined pill is started. ${ }^{14}$ This is early work, but may affect future clinical guidance.

It has been asserted recently that UPA is more costeffective than LNG. ${ }^{15}{ }^{16}$ However, this result is obtained only in theoretical models, not in clinical practice. At a population level, the impact of EC has been disappointing, and Trussell cautions against the 'overselling' of oral EC agents as being able to reduce unintended pregnancy and abortion rates overall or to be cost-effective, ${ }^{17}$ suggesting that emphasis should be placed upon the efficacy for the individual where the different effectiveness of the various methods can affect outcome.

In the end it is for the woman to make an informed choice as to the method of EC she chooses and many factors may come into play.

\section{CONCLUSIONS}

There was a significant increase in women using UPA for EC compared with our previous study. This was particularly so among those wishing to use the condom for continuing contraception. Women choosing LNG were more likely to be quick starting hormonal contraception or continuing a hormonal method. We agree with the view of others who suggest that detailed attention to continuing contraception following EC may be one factor in the prevention of unwanted pregnancy at an individual level.

It is possible that currently women choose LNG over UPA as the length of time for the use of extra contraceptive precautions is shorter. If recent work is further supported, and such use is found to be unnecessary, the provision of UPA might rise. Also, its use may increase in services that do not use quick starting. This issue should be kept under review.

Acknowledgements The authors thank Paul Dudley for efficiently retrieving the data, and the registered practitioners and doctors at their service who have so professionally embraced all of the issues in this changing emergency contraception era.

Funding This work was supported in part by the Eunice Kennedy Shriver National Institute of Child Health and Human Development grant for Infrastructure for Population Research at Princeton University (Grant R24HD047879 awarded to James Trussell).

Competing interests Aisling Baird received an honorarium from HRA Pharma for presenting these data at a UK national conference.

Provenance and peer review Not commissioned; externally peer reviewed.

\section{REFERENCES}

1 Baird AS. Use of ulipristal acetate, levonorgestrel and the copper-intrauterine device for emergency contraception following the introduction of new FSRH guidelines. J Fam Plann Reprod Health Care 2013;39:264-269.

2 Faculty of Sexual \& Reproductive Healthcare Clinical Effectiveness Unit. Emergency Contraception. August 2011, updated January 2012. http://www.fsrh.org/pdfs/ CEUguidanceEmergencyContraception11.pdf [accessed 12 June 2013].

3 Brache V, Cochon L, Deniaud M, et al. Ulipristal acetate prevents ovulation more effectively than levonorgestrel: analysis of pooled data from three randomized trials of emergency contraception regimens. Contraception 2013;88:611-618.

4 Glasier AF, Cameron ST, Logan SJS, et al. Ulipristal acetate versus levonorgestrel for emergency contraception: a randomised non-inferiority trial and meta-analysis. Lancet 2010;375:555-562.

5 Cheng L, Chey Y, Gülmezoglu AM. Interventions for emergency contraception. Cochrane Database Syst Rev 2012;8:CD001324.

6 Faculty of Sexual \& Reproductive Healthcare Clinical Effectiveness Unit. Quick Starting Contraception. September 2010. http://www.fsrh.org/pdfs/CEUGuidanceQuickStarting Contraception.pdf [accessed 29 October 2013].

7 McKay RJ, Gilbert L. An emergency contraception algorithm based on risk assessment: changes in clinicians' practice and patients' choices. J Fam Plann Reprod Health Care 2013;39:201-206. 
8 Kubba A, Connolly A, Walling M, et al. Emergency contraception: towards a multidisciplinary consensus. Prim Care Women's Health J 2012:61-64.

9 Wilcox AJ, Dunson D, Baird DD. The timing of the "fertile window" in the menstrual cycle: day specific estimates from a prospective study. BMJ 2000;321:1259-1262.

10 Stirling A, Glasier A. Estimating the efficacy of emergency contraception - how reliable are the data? Contraception 2002;66:19-22.

11 Glasier A. Emergency contraception: clinical outcomes. Contraception 2013;87:309-313.

12 Rodriguez MI, Curtis KM, Gaffield ML, et al. Advance supply of emergency contraception: a systematic review. Contraception 2013:87;590-601.

13 Cameron ST, Gordon R, Glasier A. The effect of making emergency contraception free of charge. Contraception 2012;86:366-369.
14 Cameron S, Gemzell-Danielsson KG, Klipping C, et al. Ulipristal acetate (UPA) vs levonorgestrel (LNG) and quick start contraception. Eur J Contracept Reprod Heath Care 2013; 18(Suppl. 1):ES03-2, p45.

15 Bayer LL, Edelman AB, Caughy AB, et al. The price of emergency contraception in the United States: what is the cost-effectiveness of ulipristal acetate versus single dose levonorgestrel? Contraception 2013;87: 385-390.

16 Thomas CM, Schmid R, Cameron S. Is it worth paying more for emergency hormonal contraception? The cost effectiveness of ulipristal acetate versus levonorgestrel $1.5 \mathrm{mg}$. J Fam Plann Reprod Healthcare 2010;36:197-201.

17 Trussell J. High hopes versus harsh realities: the population impact of ECPs. Eur J Contracept Reprod Heath Care 2013; 18(Suppl. 1):ES03-4, PS46.

\section{FACULTY OF SEXUAL \& REPRODUCTIVE HEALTHCARE MEMBERSHIP EXAMINATION}

The Membership Examination (MFSRH) consists of:

\section{Part 1 Single Best Answer paper (SBA)}

The London-based examination is held annually in April and October. Applications for the Friday $\mathbf{1 6}$ 0ctober $\mathbf{2 0 1 5}$ examination must be received by 1 July 2015. The syllabus for the Part 1 examination is on the FSRH website.

\section{Evidence Based Commentary (EBC)}

Candidates can view the released topic and candidate guidance notes for EBC on the Faculty website. There is an absolute deadline of $\mathbf{3 1}$ August 2015 to submit the EBC on this topic. You can submit an EBC before applying to enter the Part 1 examination.

\section{- Part 2 Examination (CRQ, EMQ, OSCE)}

Applications for the MFSRH Part 22016 (date and venue to be confirmed) must be received by 10 January 2016.

Further information and the Part 2 Syllabus are on the FSRH website. The qualification is subject to re-certification every 5 years. For the current MFSRH Examination Regulations, information on all components of the MFSRH examination and application forms, please visit the Faculty of Sexual and Reproductive Healthcare website: www.fsrh.org (Examinations) or contact Abigail Wright: abigail@fsrh.org 